\title{
Fluorescent Nanofilms for Nitroaromatic Explosives Detection-Promises and Challenges
}

\section{Linjuan Guo, Baiyi Zu and Xincun Dou*}

Laboratory of Environmental Science and Technology, Xinjiang Technical Institute of Physics and Chemistry, Chinese Academy of Sciences, China

"Corresponding author: Xincun Dou, Laboratory of Environmental Science and Technology, Xinjiang Technical Institute of Physics and Chemistry, Chinese Academy of Sciences, Urumqi 830011, China, Fax: +86-991-3838957; Tel: +86-991-3677875; E-mail: xcdou@ms.xjb.ac.cn

Received date: March 5, 2014, Accepted date: March 10, 2014, published date: March 20, 2014

Copyright: @2014 Xincun Dou, et al. This is an open-access article distributed under the terms of the Creative Commons Attribution License, which permits unrestricted use, distribution, and reproduction in any medium, provided the original author and source are credited.

\section{Editorial}

Fast and sensitive detection of nitro-aromatic explosives is of vital importance for finding hidden explosives in airport luggage or mails, screening of personnel, and detection of buried landmines [1-3]. A crucial example is the detection of trace amount of 2,4,6-trinitrotoluene (TNT), which is a typical nitro-aromatic explosives [4-8]. Various TNT detection methods are currently available, such as, the fluorescence quenching method, micromechanical sensors based on micro cantilever, the electrochemical method, ion mobility s pectroscopy (IMS), the surface-enhanced Raman spectroscopy (SERS) and semiconductor based gas sensors [7,9-23]. Among the above-mentioned methods used for TNT detection, the fluorescence quenching method is considered to be the most effective tool for sensing nitro-aromatic explosives in recent years owing to its high sensitivity, convenience, easy visualization, and short response $t$ ime for detection [24].

Much attention has been paid to the development of novel fluorescence materials for explosives detection [2,7,15,25]. Metal organic frameworks (MOFs), fluorescent conjugated polymer, and fluorescent fibers are three typical detecting materials based on fluorescence quenching method [2,11,26-28]. For MOFs, detectable changes in luminescence along with tailorable porosity and a high surface area make MOFs excellent candidates for detecting TNT [2]. Fluorescent conjugated polymers have an advantage over small fluorescent molecules in excition migration along the polymer chains, which could amplify signal like "molecular wire" [26-28]. This advantage leads to the rapid development of fluorescent conjugated polymers in explosives sensing. From 1995-2007, Swagers' group continuously demonstrated the amplification possibility with molecular wires for chemical sensors [29-34]. Based on their work, commercialized product (Fido NXT) which can detect TNT at part per quadrillion (ppq) levels has been applied in war zones of Afghanistan and Iraq [35]. In China, based on fluorescent conjugated polymers, Cheng and co-workers also explored trace explosives detector (SIM series) which could detect explosives, such as nitro-aromatic explosives and plastic explosives at part per trillion (ppt) levels in 2007 [36]. Although significant advances have been achieved, the sensing performance of fluorescent conjugated polymers depends highly on the film thickness due to the slow diffusion of analyte vapors in the non-porous rigid conjugated polymer films [30,37]. And the synthesis process of conjugated polymers is complex [38].

In this regard, electrospinning technology which is a simple and cost-effective approach to prepare nanofibrous films has been developed very rapidly in recent years [37]. Guangtao Li pioneered electrospun fibrous membranes for TNT detection, and the quenching efficiency was comparable to that of conjugated polymer-based TNT sensors [11]. Feng Liu fabricated a novel electrospun fluorescence film in which porous structure and amine modification were introduced to improve the sensitivity for detection of nitro-aromatic explosives [39]. Yu Lei synthesized a novel electrospun fluorescent nanofibrous membrane with a function like "molecular wires", leading to ultrasensitive and fast response to 2, 4-dinitrotoluene (DNT) [37].

Electrospinning is a promising method to prepare nanofibrous films, and there are several attractive aspects of this technique over others. First, the highly inherent porosity and the large surface-to-volume ratio of nanofibers. On one hand, the diffusion of explosives vapor could be greatly promoted and both of the sensitivity and the response time could be improved. On the other hand, more explosive molecules could be adsorbed on the surface of nanofibers and the quenching efficiency could be further improved [25]. Second, the simple, cost-effective and environment-friendly approach for fabricating nanofibrous films. Compared to most of the multi-step synthesis processes of fluorescent conjugated polymers, which severely restrict their practical applications, the synthesis of nanofibrous films is a one-step process and it meets practical production [38]. Third, the flexible sensor, which is reusable and light weight could be expected for smart sensing applications [40].

However, the application of nanofibrous films in practical detection of explosives confronts with some challenges. First, how to achieve the goal of specific detection towards TNT using nanofibrous films? The molecular imprinting technique (MIT) is a method to prepare polymers with recognition sites having a predetermined selectivity for given molecules [41]. It would be a promising method if MIT could be applied into nanofibrous films without damaging the films. Second, how to realize the detection of explosives in liquid solvent since the morphology of nanofibrous films is prone to change or destroy. It is suggested to select a suitable polymer which has good mechanical properties. Furthermore, how to obtain more effective and sensitive fluorescence quenching? Last but not least, the response time, which is also a vital factor to evaluate the sensing performance and for practical application.

In conclusion, the design and synthesis of flexible, porous fluorescent nanofibrous films, which can specifically, rapidly and sensitively detect nitro-aromatic explosives is a promising but challenging work.

\section{References}

1. Aguilar AD, Erica SF, Mathew L, Francis T, Avi C, et al (2009) A hybrid nanosensor for TNT vapor detection. Nano Lett 10: 380-384.

2. Sanjog SN, Biplab J, Abhijeet KC, Soumya M, Sujit KG (2013) Highly selective detection of nitro explosives by a luminescent metal-organic framework. Angew Chem Int Ed 125: 2953-2957. 
3. Wang D, Sun H, Chen A, Sei-Hum J, Alex K-Y J, et al. (2012) Chemiresistive response of silicon nanowires to trace vapor of nitro explosives. Nanoscale 4: 2628-2632.

4. Pinnaduwage LA, Gehl A, Hedden DL, Muralidharan G, Thundat T, et al (2003) Explosives: A microsensor for trinitrotoluene vapour. Nature 425 474.

5. Guo LJ, Zu BY, Yang Z, Cao HY, Zheng XF, et al (2014) APTS and rGO cofunctionalized pyrenated fluorescent nanonets for representative vapor phase nitroaromatic explosive detection. Nanoscale 6: 1467-1473.

6. Lan AJ, Li KH, Wu HH, David HO, homas JE, et al (2009) A luminescent microporous metal-organic framework for the fast and reversible detection of high explosives. Angew Chem Int Ed 48: 2334-2338.

7. He G, Zhang GF, Lü FT, Fang Y (2009) Fluorescent film sensor for vaporphase nitroaromatic explosives via monolayer assembly of oligo (diphenylsilane) on glass plate surfaces. Chem Mater 21: 1494-1499.

8. Zu BY, Guo YN, Dou XC (2013) Nanostructure-based optoelectronic sensing of vapor phase explosives-a promising but challenging method. Nanoscale 5: 10693-10701.

9. Hughes AD, Glenn LC, Patrick AD, Ellington A, Eric VA (2008) A pattern recognition based fluorescence quenching assay for the detection and identification of nitrated explosive analytes. Chem Eur J 14: 1822-1827.

10. Naddo T, Che YK, Zhang W, Balakrishnan K, Yang XM, et al (2007) Detection of explosives with a fluorescent nanofibril film. J Am Chem Soc 129: 6978-6979.

11. Tao SY, Li GT, Yin JX (2007) Fluorescent nanofibrous membranes for trace detection of TNT vapor. J Mater Chem 17: 2730-2736.

12. Zhang SJ, Lü FT, Gao LN, Ding LP, Fang Y (2007) Fluorescent sensors for nitroaromatic compounds based on monolayer assembly of polycyclic aromatics. Langmuir 23: 1584-1590.

13. Meaney MS, McGuffin VL (2008) Luminescence-based methods for sensing and detection of explosives. Anal Bioanal Chem 391: 2557-2576.

14. Burattini S, Colquhoun HM, Greenland BW, Hayes W, Wade M (2009) PyreneFunctionalised, Alternating Copolyimide for Sensing Nitroaromatic Compounds. Macromol Rapid Commun 30: 459-463.

15. He G, Yan N, Yang JY, Wang HY, Ding LP, et al (2011) Pyrene-Containing Conjugated Polymer-Based Fluorescent Films for Highly Sensitive and Selective Sensing of TNT in Aqueous Medium. Macromolecules 44: 4759-4766.

16. Spitzer D, Cottineau T, Piazzon N, Josset S, Schnell F, et al (2012) BioInspired Nanostructured Sensor for the Detection of Ultralow Concentrations of Explosives. Angew Chem Int Ed 51: 5334-5338.

17. Raorane D, Lim S-HS, Majumdar A (2008) Nanomechanical assay to investigate the selectivity of binding interactions between volatile benzene derivatives. Nano Lett 8: 2229-2235.

18. Guo SJ, Dong SJ (2011) Graphene and its derivative-based sensing materials for analytical devices. J Mater Chem 21: 18503-18516.

19. Salinas Y, Martínez-Máñez R, Marcos MD, Sancenón F, Costero AM, et al (2012) Optical chemosensors and reagents to detect explosives.Chem Soc Rev 41: 1261-1296.

20. Cheng SS, Dou J, Wang WG, Chen C, Lei H, et al (2012) Dopant-Assisted Negative Photoionization Ion Mobility Spectrometry for Sensitive Detection of Explosives. Anal Chem 85: 319-326.

21. Ehlert, S, Walte A, Zimmermann R (2013) Ambient Pressure Laser Desorption and Laser-Induced Acoustic Desorption Ion Mobility Spectrometry Detection of Explosives. Anal Chem 85: 11047-11053.
22. Chou A, Jaatinen E, Buividas R, Seniutinas G, Juodkazis S, et al (2012) SERS substrate for detection of explosives. Nanoscale 4: 7419-7424.

23. Piorek BD, Lee SJ, Moskovits M, Meinhart CD (2012) Free-surface microfluidics/surface-enhanced raman spectroscopy for real-time trace vapor detection of explosives. Anal Chem 84: 9700-9705.

24. Shanmugaraju S, Joshi SA, Mukherjee PS (2011) Fluorescence and visual sensing of nitroaromatic explosives using electron rich discrete fluorophores. J Mater Chem 21: 9130-9138.

25. Che YK, Gross DE, Huang HL, Yang DJ, Yang XM, et al (2012) DiffusionControlled Detection of Trinitrotoluene: Interior Nanoporous Structure and Low Highest Occupied Molecular Orbital Level of Building Blocks Enhance Selectivity and Sensitivity. J Am Chem Soc 134: 4978-4982.

26. Swager TM (1998) The molecular wire approach to sensory signal amplification. Acc Chem Res 31: 201-207.

27. Kim Y, Whitten JE, Swager TM (2005) High ionization potential conjugated polymers. J Am Chem Soc 127: 12122-12130.

28. Yang Y, Turnbull GA, Samuel ID (2010) Sensitive explosive vapor detection with polyfluorene lasers. Adv Funct Mater 20: 2093-2097.

29. Zhou Q, Swager TM (1995) Fluorescent chemosensors based on energy migration in conjugated polymers: The molecular wire approach to increased sensitivity. J Am Chem Soc 117: 12593-12602.

30. Yang J-S, Swager TM (1998) Porous shape persistent fluorescent polymer films: an approach to TNT sensory materials. J Am Chem Soc 120: 5321-5322.

31. Yang J-S, Swager TM (1998) Fluorescent porous polymer films as TNT chemosensors: electronic and structural effects. J Am Chem Soc 120: 11864-11873.

32. McQuade DT, Hegedus AH, Swager TM (2000) Signal amplification of a "turn-on" sensor: Harvesting the light captured by a conjugated polymer. J Am Chem Soc 122: 12389-12390.

33. Thomas SW, Amara JP, Bjork RE, Swager TM (2005) Amplifying fluorescent polymer sensors for the explosives taggant 2, 3-dimethyl-2, 3dinitrobutane (DMNB). Chem Commun 36: 4572-4574.

34. homas SW, Swager TM (2006) Trace Hydrazine Detection with Fluorescent Conjugated Polymers: A Turn-On Sensory Mechanism. Adv Mater 18: 1047-1050.

35. Thomas SW, Joly GD, Swager TM (2007) Chemical sensors based on amplifying fluorescent conjugated polymers. Chem Rev 107: 1339-1386.

36. Cheng JG, He QG, Feng SL, Cao HM, (2007) Chinese Patent (200710045607.1).

37. Wang Y, La A, Ding Y, Liu YX, Lei Y (2012) Novel Signal-Amplifying Fluorescent Nanofibers for Naked-Eye-Based Ultrasensitive Detection of Buried Explosives and Explosive Vapors. Adv Funct Mater 22: 3547-3555.

38. Lv YY, Xu W, Lin FW, Wu J, Xu ZK (2013) Electrospun Nanofibers of Porphyrinated Polyimide for the Ultra-sensitive Detection of Trace TNT. Sens Actuators, B 184: 205-211.

39. Yang YF, Wang HM, Su K, Long YY, Peng Z, Li N, Liu F (2011) A facile and sensitive fluorescent sensor using electrospun nanofibrous film for nitroaromatic explosive detection.J Mater Chem 21: 11895-11900.

40. Segev-Bar M, Haick H (2013) Flexible Sensors based on Nanoparticles. ACS Nano 7: 8366-8378.

41. Mosbach K, Ramström O (1996) The emerging technique of molecular imprinting and its future impact on biotechnology. Nat Biotechnol 14: 163-170. 